\title{
A Double-blind Randomized Controlled Trial for the Effects of Dry Needling on Upper Limb Dysfunction in Patients with Stroke
}

\author{
Zahra Tavakol, MD
}

Sports Medicine Research Center, Neuroscience Institute, Tehran University of Medical Sciences, Tehran, Iran

Ardalan Shariat*, PhD

Sports Medicine Research Center, Neuroscience Institute, Tehran University of Medical Sciences, Tehran, Iran

\section{Noureddin Nakhostin Ansari, PhD}

Sports Medicine Research Center, Neuroscience Institute, Tehran University of Medical Sciences, Tehran, Iran

\section{Shima Ghannadi, MD}

Sports Medicine Research Center, Neuroscience Institute, Tehran University of Medical Sciences, Tehran, Iran

\section{Roshanak Honarpishe, PhD}

Dept. of Physiotherapy, School of Rehabilitation, Tehran University of Medical Sciences, Tehran, Iran

\section{Jan Dommerholt, DPT}

Bethesda Physiocare, Bethesda, MD, USA; Myopain Seminars, Bethesda, MD, USA

\section{Pardis Noormohammadpour, MD}

Sports Medicine Research Center, Neuroscience Institute, Tehran University of Medical Sciences, Tehran, Iran

\section{Lee Ingle, PhD}

Dept, of Sport, Health \& Exercise Science, University of Hull, Kingston-upon-Hull, United Kingdom

*Corresponding Author: Ardalan Shariat, Sports Medicine Research Center, Neuroscience Institute, Tehran University of Medical Science, Tehran, Iran. E-mail: ardalansh2002@gmail.com. 
ABSTRACT: Spasticity is one of the main complications of a stroke. This double-blind, randomized controlled trial aimed to compare the result of three sessions of dry needling (DN) versus sham DN on the affected upper limbs in post-stroke survivors. We recruited 24 patients (age $57.0 \pm 9.6$ years; male $71 \%$ ). Patients were randomly allocated to two groups: a DN group and a sham DN group. The primary outcome measures were the Modified Modified Ashworth Scale (MMAS) and the Box and Block Test (BBT). Secondary outcome measures included active and passive wrist range of motion (AROM and PROM). All assessments were measured at baseline, immediately after the last session of the intervention, and one month later. Patients in the DN group had improved upper limb spasticity and passive wrist range of motion compared to control group $(P<0.05)$. There were no between-group differences in other outcome measures $(P>0.05)$. Dry needling is a useful method for improving muscle spasticity in the upper limbs of patients with stroke.

Keywords: Stroke; Spasticity; Dry needling; Sham dry needling

\section{INTRODUCTION}

Stroke is the second most common cause of death (Lozano et al., 2012) and one of the three leading causes of disability-adjusted life-years (DALYs) across the world (Murray et al., 2013). Despite the reduction in mortality rates for stroke over the past two decades, the global disease burden rate has increased (Feigin et al., 2014). In Iran, , there is a higher stroke incidence than in Western countries (Feigin et al., 2014; Azarpazhooh et al., 2010).

If not controlled, spasticity can result in MUSCLE contracture leading to mobility impairments (Ghaffari et al., 2019). Spasticity contributes to functional disability, ADL dependency, and a diminished quality of life (Sc et al., 2018; Duncan et al., 2003). Hence, the treatment of spasticity may restore active, voluntary movements and regain selective motor function, reduce the level of disability, and improve individual health levels (Zorowitz, Gillard, \& Brainin, 2013). Post-stroke spasticity affects the flexors of the upper limb, leading to upper limb hemiplegic posture and hand dysfunction (Opheim, Danielsson, Murphy, Persson, \& Sunnerhagen, 2014).

Dry needling (DN) has emerged as a relatively safe and cost-effective procedure for treating muscle spasticity (Shariat et al., 2018; Ghannadi et al., 2020). Due to the relatively high costs associated with post-stroke care (Lim et al., 2015), finding a low cost and effective method for improving post-stroke spasticity is essential. Thus, we aimed to determine the effects of DN on post-stroke spasticity. We hypothesized that post-stroke survivors receiving three sessions DN 
would gain significant improvements in upper limb spasticity, passive range of motion, and patients' function after stroke compared to control.

\section{MATERIALS AND METHODS}

\section{Design}

The study was a double-blind, randomized controlled trial. The review board of the Sports and Exercise Medicine Research Center and the Ethical Committee of Tehran University of Medical Sciences approved the study protocol. All patients signed an informed consent form before the study initiation.

\section{Participants}

The trial was conducted between August and October 2018 in the Sports Medicine Research Center, Tehran University of Medical Sciences, Iran.

The inclusion criteria were: 1) age between $18 \geq$; 2) at least six months since the stroke; 3) the first-ever stroke resulted in hemiplegia; 4) wrist flexor Modified Modified Ashworth Scale (MMAS) score $\geq 1 ; 5$ ) not taking any medications for spasticity, and 6) able to understand and follow instructions. The exclusion criteria were: 1) having any contraindication to dry needling; 2) history of neurological pain; 3) fixed muscle contracture of the affected wrist; 4) currently receiving other treatment protocols, and 5) unwillingness to participate in the study.

\section{Outcome measurements}

The primary outcome measures were the MMAS, and the Box and Block Test (BBT). Secondary outcome measures were active and passive wrist range of motion (AROM and PROM) measured at baseline (T0), immediately after the end of treatment (T1), and at onemonth follow-up (T2).

\section{Procedures}

Baseline clinical characteristics, including age, sex, body mass index (BMI), time since stroke, hemiplegic side, co-morbidities, and medication usage were recorded. Dry needling was delivered for three sessions, separated by a 48 -hours interval between sessions. An experienced physiotherapist, blinded to the patient allocation, preformed the assessments.

\section{Modified Modified Ashworth Scale}

The MMAS is a valid and reliable tool for assessing post-stroke spasticity, using a 0 to 4 scale. A recent study concluded that MMAS is an appropriate scale for clinical practice and research (Banky, Ryan, Clark, Olver, \& Williams, 2017). The Persian version of the MMAS was used, which has been validated previously in post-stroke survivors (Nakhostin Ansari et al., 2012). 


\section{Range of motion}

Active and passive wrist extension was measured in the sitting position using a standard manual goniometer.

\section{Box and block test}

Hand dexterity was assessed with the BBT. With this test, a box is divided into two opposing equal compartments. Patients were instructed to move 150 standard blocks from one compartment into the other compartment one block at a time using their affected hand. The assessor encouraged patients to perform the test as quickly as possible (Fakhari et al., 2017).

\section{Dry needling procedure}

Dry needling was performed with the patient in the supine position, the affected arm alongside the trunk, the shoulder at $45^{\circ}$ abduction, the elbow was extended, and the forearm in supination. Disposable sterile stainless-steel needles (size: $0.25 \mathrm{~mm}$ x $25 \mathrm{~mm}$; SMC, Seoul, Korea) were used with the fast-in and fast-out cone shape technique. Target muscles were the flexor carpi radialis (FCR) and flexor carpi ulnaris (FCU). The FCR was needled in the medial forearm $4 \mathrm{~cm}$ below and $1 \mathrm{~cm}$ medially from the midpoint of the elbow crease (Figure $1 \mathrm{~A}$ and 1B). The FCU was needled at the midpoint of the proximal third segment of a line connecting the medial epicondyle to the ulnar styloid process (Figure 1C). Each muscle was needled for 1 minute. An experienced sports medicine specialist not involved in the assessment of the patients completed the treatments. In the control group, the same protocol was carried out using a sham needle (Figure 2). All patients were instructed not to have any other treatments during the study and follow up period, including other physical therapy treatments, medications, acupuncture, or dry needling.
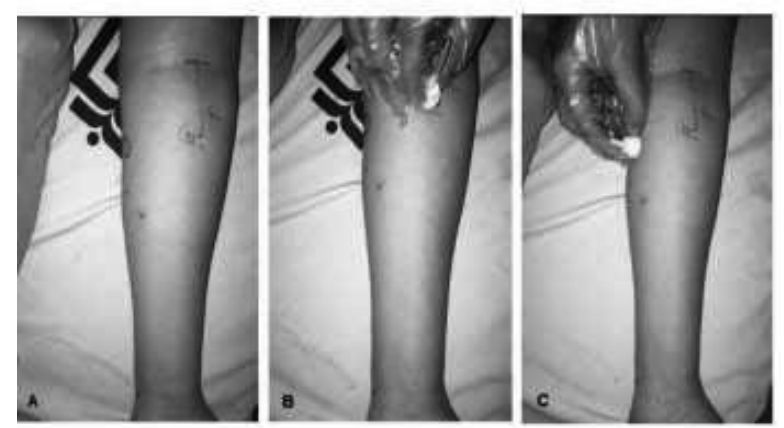

Figure 1. Location of dry needling (A). Dry needling was applied to the flexor carpi radials muscles (B) and the flexor carpi ulnaris muscles (C). 


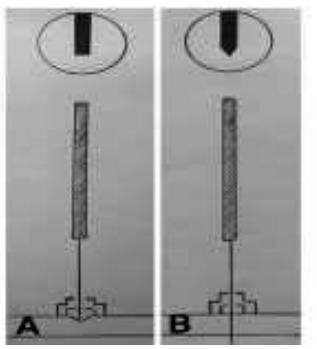

Figure 2. Real (A) and sham (B) needle.

\section{Randomization and blinding}

Patients were allocated to two groups by computer-generated randomization in blocks of 24 in a 1:1 ratio. The patients were randomized to 1 of 2 groups: a DN group and a sham DN group. A research assistant not involved in the study opened the sealed opaque envelopes and assigned the patients to their respective groups. The patients and assessor were blinded to the treatment allocation.

\section{Sample size estimates}

The estimated sample size for each group was calculated 11 (alpha=0.05; Beta 0.8 ) using data provided by Fakhari et al. (2017). With an anticipated attrition rate of $10 \%$, the number of patients in each group was increased to 12 .

\section{Statistical Analyses}

The SPSS for Windows v20 (IBM, NY, USA) was used for all statistical analyses. Dat: normality was checked by the Kolmogorov-Smirnov test. Baseline characteristics betwee groups were compared using an independent t-test and Mann-Whitney U test. Differences ove time between the groups were assessed by a $2 \times 3$ (group by time) repeated measures analysis o variance. Bonferroni post hoc adjustments were carried out where necessary with a partial eta $\left(\mathrm{y}_{p}^{2}\right)$. Partial eta ${ }^{2}$ effect sizes were interpreted as $0.25,0.40$, and $>0.40$ representing small medium, and large effect sizes (Richardson, 2011). The kruskal-wallis was used for betwee group comparison and Friedman's test was considered to test for the effects of interventions or spasticity assessed by an ordinal MMAS. A post hoc Wilcoxon Signed-Rank Test (WSRT) wa applied for paired differences between the testing time points within groups. $P$-values less tha1 0.05 were considered significant. 


\section{RESULTS}

We recruited 24 patients (age $57.0 \pm 9.6$ years; BMI $26.4 \pm 1.8$; male/female:17/7 for the study. There were no significant difference in patients' characteristics between-groups $(P>0.05)$.

\section{Modified Modified Ashworth Scale}

There was a significant differences of MMAS scores in the DN group $\left(X^{2}(2)=15.2\right.$, $p<.001)$. There was not statistically difference in time points in control group $\left(X^{2}(2)=2.00\right.$, $p=0.37$ ). A kruskal-wallis test showed that there was a statistically significant difference between two groups in post-test $(P=0.012)$.

Table-3 The Modified Modified Ashworth Scale (MMAS). Scores in both groups presented as the median.

\begin{tabular}{lllllll}
\hline & \multicolumn{3}{c}{ Intervention Group (n=12) } & \multicolumn{3}{c}{ Control Group (n=12) } \\
\hline MMAS & Pre-test & Post-test & Follow-up & Pre-test & Post-test & Follow-up \\
$\mathbf{0}$ & 0 & $5(41.7 \%)$ & $1(8.3 \%)$ & 0 & 0 & 0 \\
$\mathbf{1}$ & $6(50 \%)$ & $3(25 \%)$ & $7(58.3 \%)$ & $3(25 \%)$ & $4(33.3 \%)$ & $4(33.3 \%)$ \\
$\mathbf{2}$ & $3(25 \%)$ & $4(33.3)$ & $2(16.7 \%)$ & $5(41.7 \%)$ & $4(33.3 \%)$ & $4(33.3 \%)$ \\
$\mathbf{3}$ & $3(25 \%)$ & 0 & $2(16.7 \%)$ & $4(33.3 \%)$ & $4(33.3 \%)$ & $4(33.3 \%)$ \\
$\mathbf{4}$ & 0 & 0 & 0 & 0 & 0 & 0 \\
\hline
\end{tabular}

\section{BBT}

The group-by-time interaction $\left(P=0.187, \mathrm{~F}(2,44)=1.742, \mathrm{\eta}_{p}^{2}=.073\right)$ for BBT was not significant. A time effect $\left(p=.421, \mathrm{~F}(2,44)=.883, \mathrm{\eta}_{p}^{2}=.039\right)$ was also not evident. Between groups compression showed no significant changes $(P>0.05)$.

\section{PROM for wrist movement}

A significant group-by time interaction $\left(P<0.001, \mathrm{~F}(2,44)=72.315, \mathrm{\eta}_{p}^{2}=.767\right)$ showed that the two groups responded differently to the intervention. A significant time effect $(P<0.001, \mathrm{~F}(2$, 44) $=69.234, \mathrm{\eta}_{p}^{2}=.759$ ) also occurred (Table 1).. Between group compression showed significant differences $(P>0.05)$. 


\section{AROM for wrist movement}

The group-by-time interaction $\left(\mathrm{P}=0.145, \mathrm{~F}(1.945,28.336)=2.193, \mathrm{\eta}_{p}^{2}=.011\right)$ for $\mathrm{AROM}$ was not evident. No significant time effect $\left(p=0.311, \mathrm{~F}(1.288,28.336)=6.468, \mathrm{\eta}_{p}^{2}=.145\right)$ was found. Between groups compression showed no significant differences $(P>0.05)$.

Table-1. Changes in wrist extension range of motion and hand dexterity in experimental and control groups ( $\mathrm{n}=24)$ at baseline (T0), after completion of the intervention (T1), and 4 weeks later (T2).

\begin{tabular}{|c|c|c|c|c|c|c|c|c|c|}
\hline \multirow{2}{*}{$\begin{array}{l}\text { Variab } \\
\text { les }\end{array}$} & \multicolumn{3}{|c|}{ Experimental Group $(\mathrm{n}=12)$} & \multicolumn{3}{|c|}{ Control Group $(\mathrm{n}=12)$} & \multirow{2}{*}{$\begin{array}{c}\text { Tim } \\
\text { e } \\
\text { Eff } \\
\text { ect } \\
(P- \\
\text { val } \\
\text { ue })\end{array}$} & \multirow{2}{*}{$\begin{array}{c}\text { Betw } \\
\text { een } \\
\text { group } \\
(P- \\
\text { value } \\
)\end{array}$} & \multirow{2}{*}{$\begin{array}{c}\text { Group } \\
\text { by } \\
\text { Time } \\
\text { Interac } \\
\text { tion } \\
(P- \\
\text { value })\end{array}$} \\
\hline & $\begin{array}{l}\text { Pre } \\
\text { test(T0) }\end{array}$ & $\begin{array}{l}\text { Post } \\
\text { test(T1) }\end{array}$ & $\begin{array}{l}\text { Follow- } \\
\text { up(T2) }\end{array}$ & $\begin{array}{l}\text { Pre } \\
\text { test(T0) }\end{array}$ & $\begin{array}{l}\text { Post } \\
\text { test(T1) }\end{array}$ & $\begin{array}{l}\text { Follow- } \\
\text { up(T2) }\end{array}$ & & & \\
\hline $\begin{array}{l}\text { PRO } \\
\text { M } \\
\text { (degre } \\
\text { es) }\end{array}$ & $\begin{array}{l}47.75 \pm 1 \\
9.09\end{array}$ & $\begin{array}{l}93.25 \pm 3 . \\
98\end{array}$ & $\begin{array}{l}94.08 \pm 4 . \\
09\end{array}$ & $\begin{array}{l}63.58 \pm 2 \\
2.03\end{array}$ & $\begin{array}{l}64.08 \pm 2 \\
2.23\end{array}$ & $\begin{array}{l}64.18 \pm 2 \\
0.98\end{array}$ & $\#$ & 0.040 & $\#$ \\
\hline $\begin{array}{l}\text { ARO } \\
\text { M } \\
\text { (degre } \\
\text { es) }\end{array}$ & $\begin{array}{l}23.83 \pm 2 \\
7.88\end{array}$ & $\begin{array}{l}26.16 \pm 2 \\
7.59\end{array}$ & $\begin{array}{l}25.83 \pm 2 \\
7.59\end{array}$ & $\begin{array}{l}14.00 \pm 1 \\
5.77\end{array}$ & $\begin{array}{l}14.50 \pm 1 \\
6.12\end{array}$ & $\begin{array}{l}14.75 \pm 1 \\
6.12\end{array}$ & $\begin{array}{l}0.31 \\
1\end{array}$ & 0.249 & 0.145 \\
\hline BBT & $\begin{array}{l}6.34 \pm 9.2 \\
8\end{array}$ & $\begin{array}{l}7.00 \pm 9.4 \\
2\end{array}$ & $\begin{array}{l}6.84+9.5 \\
4\end{array}$ & $\begin{array}{l}3.41 \pm 3.0 \\
5\end{array}$ & $\begin{array}{l}3.34 \pm 2.7 \\
4\end{array}$ & $\begin{array}{l}3.25 \pm 2.7 \\
7\end{array}$ & $\begin{array}{l}0.42 \\
1\end{array}$ & 0.244 & 0.187 \\
\hline
\end{tabular}

Note: Mean \pm SD for outcome measurements. AROM: Active Range of Motion; PROM: Passive Range of Motion; BBT: Box and Block Test. $\# P<.001$

\section{DISCUSSION}

Our study examined the effects of three sessions of dry needling vs. sham needling on spasticity of post-stroke survivors. We found improvements in upper limb spasticity (MMAS) and wrist PROM compared to control. The improvements remained in wrist passive range of motion one month later. 
A decrease in muscle spasticity is similar to findings from other studies with stroke (Ansari, Naghdi, Fakhari, Radinmehr, \& Hasson, 2015; Salom-Moreno et al., 2014). The possible mechanisms are still unclear. Central modulation may lead to changes in the spinal reflex's excitability with activation of the noxious inhibitory control system. Furthermore, biomechanical changes in the needled muscles could consider as another reason (Ansari et al., 2015; Fakhari et al., 2017; Gallego \& del Moral, 2007). Increased wrist PROM could be due to the localized biomechanical changes in contractile elements in agreement with previous studies (Ansari et al., 2015; Fakhari et al., 2017; Gallego \& del Moral, 2007).

Spasticity is considered one of the most significant factors that interfere with motor recovery (Van Kuijk, Geurts, Bevaart, \& Van Limbeek, 2002). Improvements in spasticity may lead to functional recovery (Francis et al., 2004). Fakhari et al. showed a negative association between spasticity and increased AROM following one session of DN (Fakhari et al., 2017). The authors proposed that repeated task-oriented exercises combined with DN treatments may lead to better improvements in hand function (Fakhari et al., 2017). We did not find any evidence of increased AROM. However, functional impairment in the post-stroke upper limbs may be due to a neural drive down-regulation to skeletal muscles, and reduced connectivity to the corticospinal system (Bowden, Taylor, \& McNulty, 2014). Sanchez-Mila et al. showed no improvement in motor function after one session of DN in the lower extremity; moreover, they reported increased passive range of motion and decreased spasticity (Sánchez-Mila et al., 2018). Fakhari reported a significant positive relation between BBT and AROM, leading the authors to conclude that wrist AROM is an important factor for improving hand function in post-stroke survivors (Fakhari et al., 2017). In our study, BBT showed no improvement, which is understandable since we did not observe any increase in AROM following three sessions of DN.

\section{STUDY LIMITATION}

The study is not without limitations. Our intervention included three sessions of DN, and it is conceivable that additional treatment sessions could lead to more significant and longerlasting results. Hence, further studies with additional sessions are required. Combining DN with other therapies, e.g., exercises, warrant further investigations to determine if combined treatments improve outcomes. Finally, participants were not follow-up for long term.

\section{CONCLUSION}

Three dry needling sessions appear to be a useful method for improving muscle passive range of motion in the upper limb in patients following stroke. 


\section{ACKNOWLEDGMENT}

Funding: This study was supported by the Sports Medicine Research Center, Neuroscience Institute, Tehran University of Medical Sciences.

Ethical Approval: Tehran University approved this study of Medical Sciences (IR.TUMS.VCR.REC.1397.721).

Declaration of Conflicting Interests: The authors declared no potential conflicts of interest concerning the research, authorship, and publication of this article.

\section{REFERENCES}

Ansari, N. N., Naghdi, S., Fakhari, Z., Radinmehr, H., \& Hasson, S. (2015). Dry needling for the treatment of poststroke muscle spasticity: a prospective case report. NeuroRehabilitation, $36(1), 61-65$.

Azarpazhooh, M. R., Etemadi, M. M., Donnan, G. A., Mokhber, N., Majdi, M. R., GhayourMobarhan, M., ... Panahandeh, M. (2010). Excessive incidence of stroke in Iran: evidence from the Mashhad Stroke Incidence Study (MSIS), a population-based study of stroke in the Middle East. Stroke, 41(1), e3-e10.

Banky, M., Ryan, H. K., Clark, R., Olver, J., \& Williams, G. (2017). Do clinical tests of spasticity accurately reflect muscle function during walking: a systematic review. Brain Injury, 31(4), 440-455.

Bowden, J. L., Taylor, J. L., \& McNulty, P. A. (2014). Voluntary activation is reduced in both the more-and less-affected upper limbs after unilateral stroke. Frontiers in Neurology, 5, 239.

Duncan, P., Studenski, S., Richards, L., Gollub, S., Lai, S. M., Reker, D., ... Rigler, S. (2003). Randomized clinical trial of therapeutic exercise in subacute stroke. Stroke, 34(9), 21732180 .

Fakhari, Z., Ansari, N. N., Naghdi, S., Mansouri, K., \& Radinmehr, H. (2017). A single group, pretest-posttest clinical trial for the effects of dry needling on wrist flexors spasticity after stroke. NeuroRehabilitation, 40(3), 325-336.

Feigin, V. L., Forouzanfar, M. H., Krishnamurthi, R., Mensah, G. A., Connor, M., Bennett, D. A., ... Truelsen, T. (2014). Global and regional burden of stroke during 1990-2010: findings from the Global Burden of Disease Study 2010. The Lancet, 383(9913), 245-255.

Francis, H. P., Wade, D. T., Turner-Stokes, L., Kingswell, R. S., Dott, C. S., \& Coxon, E. A. (2004). Does reducing spasticity translate into functional benefit? An exploratory metaanalysis. Journal of Neurology, Neurosurgery \& Psychiatry, 75(11), 1547-1551.

Gallego, P. H., \& del Moral, O. M. (2007). A case study looking at the effectiveness of deep dry needling for the management of hypertonia. Journal of Musculoskeletal Pain, 15(2), 55-60.

Ghaffari, M. S., Shariat, A., Honarpishe, R., Hakakzadeh, A., Cleland, J. A., Haghighi, S., \& Barghi, T. S. (2019). Concurrent Effects of Dry Needling and Electrical Stimulation in the Management of Upper Extremity Hemiparesis. Journal of acupuncture and meridian studies, 12(3), 90-94. 
Ghannadi, S., Shariat, A., Ansari, N. N., Tavakol, Z., Honarpishe, R., Dommerholt, J., ... \& Ingle, L. (2020). The Effect of Dry Needling on Lower Limb Dysfunction in Poststroke Survivors. Journal of Stroke and Cerebrovascular Diseases, 104814.

Lim, S. M., Yoo, J., Lee, E., Kim, H. J., Shin, S., Han, G., \& Ahn, H. S. (2015). Acupuncture for spasticity after stroke: a systematic review and meta-analysis of randomized controlled trials. Evidence-Based Complementary and Alternative Medicine, 2015.

Lozano, R., Naghavi, M., Foreman, K., Lim, S., Shibuya, K., Aboyans, V., ... Ahn, S. Y. (2012). Global and regional mortality from 235 causes of death for 20 age groups in 1990 and 2010: a systematic analysis for the Global Burden of Disease Study 2010. The Lancet, 380(9859), 2095-2128.

Murray, C. J. L., Vos, T., Lozano, R., Naghavi, M., Flaxman, A. D., Michaud, C., ... Abdalla, S. (2013). Disability-adjusted life years (DALYs) for 291 diseases and injuries in 21 regions, 1990-2010: a systematic analysis for the Global Burden of Disease Study 2010. The Lancet, 380(9859), 2197-2223.

Nakhostin Ansari, N., Naghdi, S., Forogh, B., Hasson, S., Atashband, M., \& Lashgari, E. (2012). Development of the Persian version of the Modified Modified Ashworth Scale: translation, adaptation, and examination of interrater and intrarater reliability in patients with poststroke elbow flexor spasticity. Disability and Rehabilitation, 34(21), 1843-1847.

Opheim, A., Danielsson, A., Murphy, M. A., Persson, H. C., \& Sunnerhagen, K. S. (2014). Upper-limb spasticity during the first year after stroke: stroke arm longitudinal study at the University of Gothenburg. American Journal of Physical Medicine \& Rehabilitation, 93(10), 884-896.

Richardson, J. T. E. (2011). Eta squared and partial eta squared as measures of effect size in educational research. Educational Research Review, 6(2), 135-147.

Salom-Moreno, J., Sánchez-Mila, Z., Ortega-Santiago, R., Palacios-Ceña, M., TruyolDomínguez, S., \& Fernández-de-las-Peñas, C. (2014). Changes in spasticity, widespread pressure pain sensitivity, and baropodometry after the application of dry needling in patients who have had a stroke: a randomized controlled trial. Journal of Manipulative and Physiological Therapeutics, 37(8), 569-579.

Sánchez-Mila, Z., Salom-Moreno, J., \& Fernández-de-las-Peñas, C. (2018). Effects of dry needling on post-stroke spasticity, motor function and stability limits: a randomised clinical trial. Acupuncture in Medicine, acupmed-2017.

Shariat, A., Noormohammadpour, P., Memari, A. H., Ansari, N. N., Cleland, J. A., \& Kordi, R. (2018). Acute effects of one session dry needling on a chronic golfer's elbow disability. Journal of Exercise Rehabilitation, 14(1), 138.

Van Kuijk, A. A., Geurts, A. C. H., Bevaart, B. J. W., \& Van Limbeek, J. (2002). Treatment of upper extremity spasticity in stroke patients by focal neuronal or neuromuscular blockade: a systematic review of the literature. Journal of Rehabilitation Medicine, 34(2), 51-61.

Zorowitz, R. D., Gillard, P. J., \& Brainin, M. (2013). Poststroke spasticity: sequelae and burden on stroke survivors and caregivers. Neurology, 80(3 Supplement 2), S45-S52. 